\title{
Tracking the Progression \& Influence of Beta-Amyloid Plaques Using Percolation Centrality and Collective Influence Algorithm: A Study using PET Images
}

\author{
Gautam Kumar Baboo ${ }^{1}$ (), Raghav Prasad ${ }^{1}$, Pranav Mahajan ${ }^{1}$ and Veeky Baths ${ }^{1, *}$ \\ 1 C-115, Cognitive Neuroscience Lab, Department of Biological Sciences, BITS-Pilani - K.K. Birla Goa \\ Campus; \\ F. author p20130404@goa.bits-pilani.ac.in \\ S.author: f20170297@goa.bits-pilani.ac.in, \\ T. author: f20170277@goa.bits-pilani.ac.in \\ * Correspondence: veeky@goa.bits-pilani.ac.in
}

Citation: Kumar B., Gautam.;

Prasad, Raghav.; Mahajan, Pranav.;

Baths, Veeky. Percolation Centrality and Collective Influence on

Beta-amyloid plaques network.

Journal Not Specified 2021, 1, 0.

https://doi.org/

Received:

Accepted:

Published:

Publisher's Note: MDPI stays neutral with regard to jurisdictional claims in published maps and institutional affiliations.

Copyright: () 2021 by the authors. Submitted to Journal Not Specified for possible open access publication under the terms and conditions of the Creative Commons Attribution (CC BY) license (https:// creativecommons.org This preprint reportş $4.0 /)$.

\begin{abstract}
Background: Network analysis allows investigators to explore the many facets of brain networks, particularly the proliferation of disease. One of the hypotheses behind the disruption in brain networks in Alzheimer's disease is the abnormal accumulation of beta-amyloid plaques and tau protein tangles. In this study, the potential use of percolation centrality to study beta-amyloid movement was studied as a feature of given PET image-based networks; (2) Methods: The PET image-based network construction is possible using a public access database - Alzheimer's Disease Neuroimaging Initiative, which provided 551 scans. For each image, the Julich atlas provides 121 regions of interest, which are the network nodes. Besides, using the collective influence algorithm, the influential nodes for each scan are calculated; (3) Analysis of variance $(\mathrm{p}<0.05)$ yields the region of interest Gray Matter Broca's Area for PiB tracer type for five nodal metrics. In comparison, AV45: the Gray Matter Hippocampus region is significant for three of the nodal metrics. Pairwise variance analysis between the clinical groups yields five and twelve statistically significant ROIs for AV45 and PiB, capable of distinguishing between pairs of clinical conditions. Multivariate linear regression between the percolation centrality values for nodes and psychometric assessment scores reveals Mini-Mental State Examination is reliable(4) Conclusion: percolation centrality effectively ( $41 \%$ of ROIs) indicates that the regions of interest that are part of the memory, visual-spatial skills, and language are crucial to the percolation of beta-amyloids within the brain network to the other widely used nodal metrics. Ranking the regions of interest based on the collective influence algorithm indicates the anatomical areas strongly influencing the beta-amyloid network.
\end{abstract}

Keywords: Brain Mapping; P.E.T.; Neurodegenerative Disorders; Alzheimer's Disease; Graph Theory; Percolation Centrality; Collective Influence.

\section{Introduction}

Alzheimer's disease predominantly stands out when it comes to neurodegenerative diseases affecting the middle-age (early-onset Alzheimer's disease (A.D.)) and the oldage (Late-onset $\mathrm{AD}$ ) human population. Current projections are estimated to cost about 2 trillion U.S. Dollars by 2030[1] affecting 75 million individuals by the same year. The indirect costs are estimated to be about 244 billion U.S. Dollars[2]. With no sight of a cure for A.D. and with increasing cases, early diagnosis and active management are the keys to tackling this disease for now. The ability to predict the disease's progression with high accuracy helps design a suitable treatment regime at an early stage, thereby bringing the disease's management to an affordable cost range.

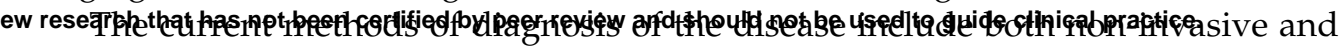
invasive techniques of investigations ranging from Positron Emission Tomography (PET) 
scans[3] or Cerebrospinal Fluid (CSF) analysis[4]. Positron Emission Tomography or PET imaging involves the use of radiopharmaceuticals such as 2-[18F], florbetapir-fluorine-18 (AV45), or 11C-Pittsburgh compound B (PiB). AV45 and PiB[5] are comparatively newer and different in terms of the image construction mechanism. Both AV45 and PiB bind to beta-amyloid but vary in their half-life. AV45 has a half-life of 109.75 minutes and PiB, 20 minutes[6]. A comparison between PiB and AV45 varies because AV45 shows uptake within the white matter regions[7].

A combination of techniques or criteria is currently employed to detect and determine the extent of dementia due to AD. Methods which include family history, psychiatric history for cognitive and behavioral changes, which is then followed by psychometric assessments such as Mini-Mental State Examination (MMSE)[8], Frontal Assessment Battery[9] and the Neuropsychiatric Inventory Questionnaire (NPIQ)[10].

The MMSE questionnaire provides assessment in five areas of cognitive function; Orientation, Attention, Memory, Language, and Visual-Spatial skills. Similarly, the NPI questionnaire provides assessment in twelve neuropsychiatric symptoms. These two questionnaires provide the classification of the patients into three clinical conditions; cognitively normal, mild cognitive impairment, and dementia due to A.D.

The application of network analysis/graph theory to anatomical neural networks has proved useful in understanding the brain connectivity[11,12](deviations under various psychological and neurological disease states. Network analysis on neuroimaging data such as EEG, MEG, fMRI, and PET scans proves to be useful to show the variation between a cognitively normal population versus other diagnostic states using various graph-theoretic metrics[13,14].

Graph metrics such as characteristic path length, clustering coefficient, modularity, and hubs have been studied and have provided insights into the brain networks of AD patients and control groups. Some studies have tried to map the progression of MCI to dementia due to $\mathrm{AD}[15,16]$; thus, network analysis and the various graph metrics have shown potential as a tool to investigate the brain networks. Network analysis on AD is a practical application wherein it describes the Alzheimer's brain network's behaviour. Connectivity analysis using fMRI and EEG data reports provides mixed responses; when comparing $\mathrm{AD}$ patients and the control group[17], there is an increase or decrease in the network's connectivity. A reduction in connectivity could explain the cortical atrophy/disruption of the network. An increase could explain the compensatory mechanism[18].

Network Analysis on PET images related to A.D. mainly revolve around learning models or are limited to tracers that focus on the metabolic networks and the associated deviations of these networks[19,20]. Other methods include applying algorithms to the raw PET images to recognize patterns to resolve differences between healthy controls and patients with neurodegeneration[21]. The use of PET imaging and lumbar puncture to determine the levels of beta amyloids in either of them beyond the normal levels is the current standard of practice for the determination of dementia due to A.D.[22,23].

To understand beta-amyloid propagation, we propose applying graph theoretic methods on PET images to understand beta-amyloid advancement. The main benefit of adding this method is that

- This does not introduce any new steps for data collection from the patient and, at the same time.

- Adds value to the existing data by computing the percolation centrality of a given node at a given time

Network topology offers insights into the evolution of the network in a clinical setting. Studying such an evolution provides a possibility to understand the weak links within Julich atlas[24-26] based region of interest(ROI) networks. Such networks' structural connectivity information might yield the source and sink of neurodegeneration with the brain architecture. 
Percolation centrality is defined as the proportion of 'percolated paths' that pass through that node; this measure quantifies the relative impact of nodes based on their topological connectivity, as well as their percolated states. In other words, it is one such graph metric that looks at the extent to which a given node within a network has percolated information or can percolate information(Figure 1). The volume of information transmitted via a given node is provided by values ranging from 0.0 to $1.0[27,28]$. Prior exploration of percolation centrality on disease networks[28-31] and percolation centrality in disease networks of the brain[32] have shown this as a promising metric for brain network investigation.

The knowledge on the application of percolation centrality on human PET-imagebased networks is scarce at present. This work aims at adding knowledge to the gap. On the other hand, collective influence provides a minimum set of nodes or regions of the interest that can transfer information or spread disease with ease with optimal spread[33] based on the optimal percolation theory. By examining the network for the minimum set of nodes, this set will provide the regions of interest within the brain that optimally move beta-amyloid, disrupting the normal functioning of the existing neural networks.

Thus, the ability to detect the disease and predict the rate of progression of the disease at an early stage is imperative. To this end, the study aims to answer two main questions:

1) Can percolation centrality measure be used to determine the percolation of beta-amyloids within the brain?

2) Can the collective influence algorithm provide a minimum set of nodes that are vital to the AD network?

\section{Materials and Methods}

\subsubsection{Patient Distribution}

Based on the tracer agents used for acquiring the PET images, each diagnostic state subset of the data set is divided into the two available tracers; AV45[34] and PiB[6]. The patients are categorized as Cognitively normal, with Mild Cognitive Impairment, or having Alzheimer's Disease (AD) based on the ADNI study's psychometric assessments. Next, the PET image is matched with the patient's diagnostic state at the time of the imaging procedure. This provided a set of observations for each type of tracer for each patient condition clinical group((Table 1). Finally, the resulting set of patients is matched with the demographic information providing 531 patients.

\subsection{Network Construction and Processing \\ 2.1.1. PET Image preprocessing}

Image preprocessing is carried out in two steps (Figure 2):

1. Combining individual frames of the PET image to form a $4 \mathrm{D}$ raw activity image. This is done using the fslmerge utility included in FSL[35].

2. The $4 \mathrm{D}$ raw activity image is converted to a $4 \mathrm{D}$ SUV image using the following formula:

$$
S U V=\frac{c_{i m g}}{c_{i n j}}
$$

where $c_{i m g}\left(\mathrm{Mbq} \mathrm{ml}^{-1}\right)$ is given by the raw activity image and $c_{i n j}=\frac{I D}{B W} . I D(\mathrm{MBq})$ is the injection dose[36], and $B W(\mathrm{~g})$ is the body weight of the patient, considering the equivalency $1 \mathrm{~g}=1 \mathrm{ml}$

3. Spatially realigning the PET frames to correct for motion. This was done using MCFLIRT.[37] The motion correction occurs with 6 DOF. The PET frames are realigned using the mean image as a template. The mean image is obtained by applying the motion correction parameters to the time series and averaging the volumes. 
4. Coregistering the 4D SUV image from subject space to MNI[38] space. This is done using FreeSurfer[39]. The image used for coregistration is the MNI152_T1_2mm_brain.

To parallelize this operation, GNU Parallel[40] is used.

\subsection{PET Image-based Network Construction}

The network is constructed using the regions of interest (ROIs) from the Julich Atlas. This atlas provides 121 ROIs, which translates to 121 nodes or vertices in the network((Figure 3). Building networks from the preprocessed images requires the 12 generation of adjacency matrices. The adjacency matrix is computed by calculating the 43 method described below.

The Bivariate Pearson correlation performs poorly in cases of "confounding" or "chain" interactions. In such cases, partial correlation measures the direct connectivity between two nodes by estimating their correlation after regressing out effects from all the other nodes in the network, hence avoiding spurious effects in network modeling. Whereas in cases of "colliding" interactions, a partial correlation may induce a spurious correlation. Thus, Sanchez-Romero and Cole have introduced a combined multiple functional connectivity method[41].

The network is constructed by computing the pairwise partial correlation values of voxel intensities in the PET images to produce an initial adjacency matrix (matrix $x_{\text {part }}$ ). A second matrix (matrix bivar $_{\text {) }}$ is constructed by computing the bivariate correlation values of voxel intensities in the PET images. Now, matrix part is modified using matrix $x_{\text {bivar }}$ as follows:

$$
\text { matrix }_{\text {part }}(i, j)= \begin{cases}0 & \text { if matrix } \\ \text { no change } & (i, j)=0 \\ \text { otherwise }\end{cases}
$$

where matrix part $(i, j)$ and matrix $_{\text {bivar }}(i, j)$ is the element at $(i, j)$ in the respective matrices. matrix part is now the combinedFC adjacency matrix that defines the network((Figure 4).

The partial correlation is calculated using the correlation between two residuals; the values are computed using $N-2$ ROIs as co-factors for every pair of ROIs[42]. The partial correlation values serve as the edge weights and constitute the values in the adjacency matrices.

Partial correlations are computed as correlation of residuals. The first order partial correlation $\left(\rho_{i j . k}\right)$ of $x_{i}$ and $x_{j}$, controlling for $x_{k}$ is given by [43].

$$
\operatorname{corr}(\operatorname{resid}(i \mid k), \operatorname{resid}(j \mid k))=\frac{c_{i j}-c_{i k} v_{k} c_{k j}}{\sqrt{v_{i}-c_{i k} v_{k} c_{k i}} \sqrt{v_{j}-c_{j k} v_{k} c_{k j}}}
$$

where $c_{i j}=\operatorname{cov}\left(x_{i}, x_{j}\right)$ and $v_{k}=\operatorname{var}\left(x_{k}\right)$ Further,

$$
\rho_{i j . k}=\frac{\rho_{i j}-\rho_{i k} \rho_{j k}}{\sqrt{1-\rho_{i k}^{2}} \sqrt{1-\rho_{j k}^{2}}}
$$

Since we are controlling for $(N-2)$ ROIs for each pair of ROIs $R O I_{i}$ and $R O I_{j}$, we calculate the $(N-2)^{t h}$ order partial correlation. This is calculated recursively as

For each $\mathrm{ROI}_{k} \in \mathbf{R O I s}$

$$
\rho_{i j . \mathbf{R O I s}}=\frac{\rho_{i j . \mathbf{R O I} \backslash\{k\}}-\rho_{i k . \mathbf{R O I} \backslash\{k\}} \rho_{k j . \mathbf{R O I s} \backslash\{k\}}}{\sqrt{1-\rho_{i k . \mathbf{R O I s} \backslash\{k\}}^{2}} \sqrt{1-\rho_{k j . \mathbf{R O I s} \backslash\{k\}}^{2}}}
$$

The base case of this recursive algorithm is given by equation 3 . The estimation of partial correlations is a computationally intensive task, mainly due to the pre-calculation 
of residuals before computing cross-correlation. And because the number of covariates is large; this calculation is done in a time-optimized manner using the $\mathrm{R}$ package ppcor[43].

Next, the adjacency matrices with set threshold; using a) data-driven threshold scheme based on Orthogonal Minimal Spanning Trees (OMSTs)[21,44,45]. Network threshold serves to remove inconsequential (or low-impact) edges and reduce the network complexity. And b) shortest path thresholding scheme [46]. This is done to compare between the two common schemes used for threshold.

The Networkx[47] Python library is used for network construction from the threshold adjacency matrices and subsequent percolation centrality computation and other graph metrics.

\subsection{Percolation Centrality Computation}

Percolation centrality is a nodal metric and is calculated for each node. The percolation centrality for each node $\mathrm{v}$ at time $\mathrm{t}$ is calculated as shown below:

$$
P C^{t}(v)=\frac{1}{(N-2)} \sum_{s \neq v \neq r} \frac{\sigma_{s, r}(v)}{\sigma_{s, r}} \frac{x_{s}^{t}}{\left[\sum x_{v}^{t}\right]-x_{v}^{t}}
$$

Where $\sigma_{s, r}$ is the number of shortest paths between nodes $s$ and $r$ pass-through node $v$, $x_{i}^{t}$ is the percolation state of node $i$ at time $t$,

$x_{i}^{t}=0$ indicates a non-percolated node and,

$x_{i}^{t}=1$ indicates a fully percolated node.

The percolation centrality value is calculated for each network using the inbuilt function of Networkx.(see supplementary data)

\subsection{Collective Influence Algorithm}

We define $G(q)$ the fraction of occupied sites (or nodes) belonging to the giant(largest) connected component. Percolation theory[48] tells us that if we choose these $q$ fraction of nodes randomly, the network undergoes a structural collapse at a certain critical fraction where the probability of existence of the giant connected component vanishes, $G=0$. The optimal percolation problem is finding the minimum fraction $q_{c}$ of nodes to be removed such that $G\left(q_{c}\right)=0$ i.e. the minimum fraction of "influencers" to fragment the network. For any fixed fraction $q<q_{c}$, we search for the configuration of removed nodes that provides the minimal non-zero giant connected component $G$. For further reading on how the problems of optimal immunization and spreading (optimal influencer problem) to the problem of minimizing the giant component of a network, i.e., optimal percolation problem, readers are encouraged to read [49].

The algorithm is on the basis that, given a network: the flow of information within the network is optimal with a minimum number of nodes that weigh heavily on the flow of information through the said network[33]. In the context of this investigation, the small sets of nodes/ROIs would prove to be vital in the movement of beta-amyloid plaques.

The core idea is that the overall functioning of a network in terms of the spread of information (or in our case, movement of beta-amyloid plaques) hinges on a specific set of nodes called influencers. This idea of finding the most influential nodes has been previously used in other contexts, for example, activating influential nodes in social networks to spread information[50] or de-activating or immunizing influential nodes to prevent large scale pandemics[30,51]. In recent applications to neuroscience, this method has been used to find nodes essential for global integration of a memory network in rodents[32]. Our work is the first to apply it to study the progression of $\mathrm{AD}$, to the best of our knowledge. In the context of this investigation, these small sets of influential nodes/ROIs would prove to be vital in the movement of beta-amyloid plaques.

With the implementation of Collective Influence (CI) algorithm, it facilitates to pinpoint the most influential nodes, more efficiently than previously known heuristic 
techniques. $\mathrm{CI}$ is an optimization algorithm that aims to find the minimal set of nodes that could fragment the network in optimal percolation, or in a sense, their removal would dismantle the network in many disconnected and non-extensive components. In percolation theory, if we remove nodes randomly, the network would undergo a structural collapse at a critical fraction where the probability that the giant connected component exists is $G=0$. The optimal percolation is an optimization problem that attempts to find the minimal fraction of influencers $q$ to achieve the result $G(q)=0$.

\subsection{Other Graph metrics}

Besides the percolation centrality measure, four other nodal metrics of a graph are calculated here. Below are the four metrics that are computed for the PET image based graphs.

\subsubsection{Betweenness Centrality}

The basic definition of betweenness centrality is defined as,

$$
C_{B}(u)=\frac{\sum_{s \neq u \neq t} \frac{\partial_{s t}(u)}{\partial_{s t}}}{(n-1)(n-2) / 2}
$$

This centrality information provides the uniqueness of it within the network. In this study it provides the regions of interest that play a vital role in the information flow in the network; the information being beta-amyloids or tau proteins accumulation in those regions.

\subsubsection{Closeness Centrality}

The closeness centrality of a node denotes how close a node is in the given network. It is inversely proportional to the farness of the node. Freeman defined the closeness centrality as,

$$
C_{c}(u)=\frac{n-1}{\sum_{\forall u, v \neq u} d(u, v)}
$$

The distance between two nodes/ROIs $\mathrm{u}$ and $\mathrm{v}$ in a network, denoted $\mathrm{d}(\mathrm{u}, \mathrm{v})$, is defined as the number of hops made along the shortest path between $u$ and $v$. In this case, lesser the hops; closer are the two ROI's and the ease with which the misformed proteins can travel.

\subsubsection{Current Flow Betweenness Centrality}

Given a source $\operatorname{ROI}(s)$ and a target $\operatorname{ROI}(t)$, the absolute current flow through edge $(i, j)$ is the quantity $A_{i, j}\left|v_{i}^{(s, t)}-v_{j}^{(s, t)}\right|$. By Kirchhoff's law the current that enters a node is equal to the current that leaves the node. Hence, the current flow $F_{i}^{(s, t)}$ through a node $i$ different from the source $s$ and a target $t$ is half of the absolute flow on the edges incident in $i$ :

$$
F_{i}^{(s, t)}=\frac{1}{2} \sum_{j} A_{i, j}\left|v_{i}^{(s, t)}-v_{j}^{(s, t)}\right|
$$

Moreover, the current flows $F_{s}^{(s, t)}$ and $F_{t}^{(s, t)}$ through both $s$ and $t$ are set to 1 , if end-points of a path are considered part of the path, or to 0 otherwise. Since the potential $v_{i}^{(s, t)}=G_{i, s}^{+}-G_{i, t}^{+}$, with $G^{+}$the generalized inverse of the graph Laplacian, the above equation can be expressed in terms of elements of $G^{+}$as follows:

$$
F_{i}^{(s, t)}=\frac{1}{2} \sum_{j} A_{i, j}\left|G_{i, s}^{+}-G_{i, t}^{+}+G_{j, t}^{+}-G_{j, s}^{+}\right|
$$


Finally, the current-flow betweenness centrality $b_{i}$ of node $i$ is the flow through $i$ averaged over all source-target pairs $(s, t)$ :

$$
b_{i}=\frac{\sum_{s<t} F_{i}^{(s, t)}}{(1 / 2) n(n-1)}
$$

\subsubsection{Eigenvector Centrality}

Eigenvector centrality is a measure of the influence a node has on a network. If a node is pointed to by many nodes (which also have high Eigenvector centrality) then that node will have high Eigenvector centrality. In this case, the ROI's are the nodes and PET images provide the intensity value for each ROI, which helps in computing the centrality values each scan. Another interpretation to the centrality measure is that it provides the list of prominent regions in the brain network hierarchy and helps in detection of localized differences between patient populations[52].

Previous work on AD patients and comparison with normal patients provides the usefulness of Eigenvector centrality[53].

\section{Statistical Analysis}

For this study the null hypothesis is that percolation centrality value does not indicate the propagation of beta-amyloids within the brain network.

To determine the impact the percolation value has over each PET scan, a comparison with the regions of interest from the brain atlas is done using the Multiple linear regression analysis.

This study is exploratory in nature, and that the multiplicity problem is significant. And implementation of multiple test procedures does not solve the problem of making valid statistical inference for hypotheses that were generated by the data. But it does assist in describing the possible mechanism.

\subsection{Pairwise Analysis of Variance}

To obtain pairwise group differences, we carry out a post prior (post hoc) analysis using scikit-posthocs package; the Student T-test pairwise gives us the respective $p$ values. The ANOVA test is performed for each node in the network with the null hypothesis that the mean percolation centrality of that node is the same across the three stages. To test the null hypothesis, Analysis of variance with significance level $(\alpha)$ of 0.05 is used.

\subsection{Error Correction}

To control for multiple comparisons of 121 nodes, the Scheffe Test and control for Experiment-wise Error Rate (EER) is carried out. It is a single-step procedure that calculates the simultaneous confidence intervals for all pairwise differences between means.

\subsection{Multivariate Linear Regression}

A correlation between the percolation centrality values for all 121 nodes and psychometric test scores - MMSE and NPIQ - is computed to identify the regions of interest that can be used as reliable predictors. Instead of performing multiple correlations across all three diagnoses, a multivariate regression analysis using regularisation techniques, wherein the features are the nodal percolation centrality values, and the target variable is the MMSE or NPIQ score. The goal is not to build a predictive model but to use it to quantify each node's influence in distinguishing between the clinical conditions for interpretation purposes. Had the purpose been building a machine learning model, it would imply the need to develop elaborate features sets (more than just percolation centrality) and utilize complex machine learning architectures (which provide less room for interpretability) 


\subsection{Regularization and Cross-Validation}

We use regularization in our multivariate linear regression (MLR) to make sure our regression model generalizes better to unseen data. Regularization is necessary to control for overfitting. Here, both Lasso regression (L1 penalty) as well as Ridge regression (L2 penalty) are tested, and both provide similar root mean squared errors (RMSE) and similar desired results. We choose Lasso with $\alpha=0.1$, for reporting our results (Figure 6). To quantify the robustness and reliability of our model, before and after regularization, we perform a leave one out cross-validation (LOOCV). We choose this cross-validation strategy because it is unbiased and better suited to our smaller sample sizes (especially in $\mathrm{PiB}$ tracer subset). We observe an improvement in validation RMSE with an increase in regularization (parameter $\alpha$ ), but we also observe that excessive penalization of weights at very high values of $\alpha$ can result in the regression model converging to the mean of the output MMSE/NPIQ scores. To take this into account, we also plot the standard deviation in predicted MMSE/NPIQ outputs and choose $\alpha=1$ for sufficient but not excessive regularization (Further details in supplementary figures).

\section{Results}

\subsection{Pairwise ANOVA}

The student $\mathrm{t}$-test is performed on the resulting five centrality values for each tracer type and clinical conditions. There was a significant effect of the beta-amyloid accumulation on the five centrality values at $\mathrm{p}<0.05$ level for the three clinical groups $[\mathrm{F}(3$, $454)=3.002$ for AV45 and F(3, 97) = 3.027 for PiB] (Table 2). A one-way between clinical groups ANOVA was conducted to compare the effect of beta-amyloid accumulation/tau protein on five centrality values in the cognitive normal, mild cognitive impairment and Alzheimer's disease patient(Table 3 \& Table 4)).

\subsubsection{Cross-Validation and Regularization}

Increasing regularization $(\alpha)$ improves the validation RMSE, making it more robust and generalize to unseen data. But at higher values of $\alpha$, it is observed that the standard deviation of predicted MMSE scores decreases to less than $<2$, irrespective of clinical condition. Which could mean that it saturates to predicting the mean MMSE value when regression weights are extremely penalized. Thereby choosing a reasonably small yet effective $\alpha$ value (less than 2), for which the validation RMSE and the standard deviation in output predicted MMSE.

\subsection{Multivariate Linear regression}

A linear regression model between the percolation centrality values for all 121 nodes and psychometric test scores - MMSE and NPIQ - is computed to identify the regions of interests that can be used as reliable predictors(Figure 4). Instead of performing multiple correlations across all three diagnoses, a multivariate regression analysis using leave one out cross validation is carried out, wherein the features are the nodal percolation centrality values and the target variable are the psychological assessment scores(Table 6 \& Table 7).

\subsection{Comparison of Threshold Schemes}

The two schemes are compared on the number of ROI's that can be considered on the basis of ANOVA analysis $(\mathrm{p} \leq 0.05)$, The Juelich atlas has five clusters; frontal, parietal, temporal, occipital lobes and the white matter regions. As well as com- paring the performance of the threshold schemes between the two tracers(Table 5). Orthogonal Minimum Spanning Tree: provides a total of 112 ROI's across the five nodal metrics. On the Basis of the tracers, 60 ROI's are obtained for AV45 and 52 for PiB. Further, the ranking of ROI on the basis of the threshold scheme is listed for the three clinical groups for the respective tracers 
medRxiv preprint doi: https://doi.org/10.1101/2020.10.12.20211607; this version posted August 3, 2021. The copyright holder for this preprint (which was not certified by peer review) is the author/funder, who has granted medRxiv a license to display the preprint in

It is made available under a CC-BY-NC-ND 4.0 International license .

Shortest Path Threshold: here a total of 66 ROI's are obtained. 31 and 35 for AV45 and PiB tracers respectively. On the basis of the collective influence algorithm the ROI's are ranked for the three clinical groups and their respective tracers.

Further, comparing the number of statistically valid(MLR) ROIs across the five centrality values with the psychometric tests; MMSE and NPI-Q is performed. This helps in comparing the function of the ROI with the assessment carried out on the test(Table 5 Also see supplementary material-Tables 11 through 18).

\subsection{Other Graph Metrics}

The Closeness Centrality provides the highest number of ROI's across overall(Table 10); 80 in total. Eigenvector Centrality provides 33 ROI's(Table 9), whereas Percolation Centrality has 24 ROI's followed by the Betweenness Centrality measure with a total of 23 ROI's and Current Flow Betweenness Centrality 19 ROI's across the two tracers(Table 8).

\subsection{Collective Influence Ranking}

The collective influence algorithm ranks the ROIs; here, the rank list is generated for the two tracers- AV45 and PiB. When a comparison of the rank is carried out between the clinical groups and tracers in the case of $\mathrm{PiB}$, the ranking increases when moving from $\mathrm{CN}$ clinical condition to $\mathrm{MCI}$, and then ranking decreases going from $\mathrm{MCI}$ to $\mathrm{AD}$. Overall the ranking increases by $50 \%$ from cognitively normal condition to Alzheimer's disease condition.

\subsection{Demographics}

On the basis of the selection criteria, 531 patients were available for this study. Of this, $48 \%$ of the females were of the Cognitively Normal group, $25 \%$ with Mild cognitive impairment, and 27\% with Alzheimer's disease.

$43 \%$ of the patients received more than 12 years of education as opposed to only $16 \%$ who received less than 12 years of education, 31\% received more than 12 years of education in the MCI group as opposed to $69 \%$ with less than 12 years of education.

$47 \%$ of the Left-handed patients were in the AD clinical group as opposed to $26 \%$ in the right-handed patients. One patient in the MCI, two in $\mathrm{CN}$, and four in $\mathrm{AD}$ groups were multilingual.

\subsection{Figures, Tables and Schemes}

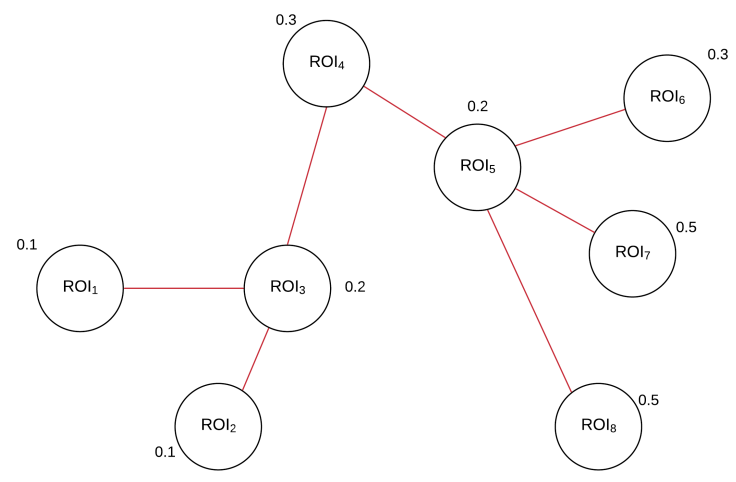

Figure 1. Generic example of a Percolation Network and Percolation Centrality 
medRxiv preprint doi: https://doi.org/10.1101/2020.10.12.20211607; this version posted August 3, 2021. The copyright holder for this preprint (which was not certified by peer review) is the author/funder, who has granted medRxiv a license to display the preprint in It is made available under a CC-BY-NC-ND 4.0 International license.

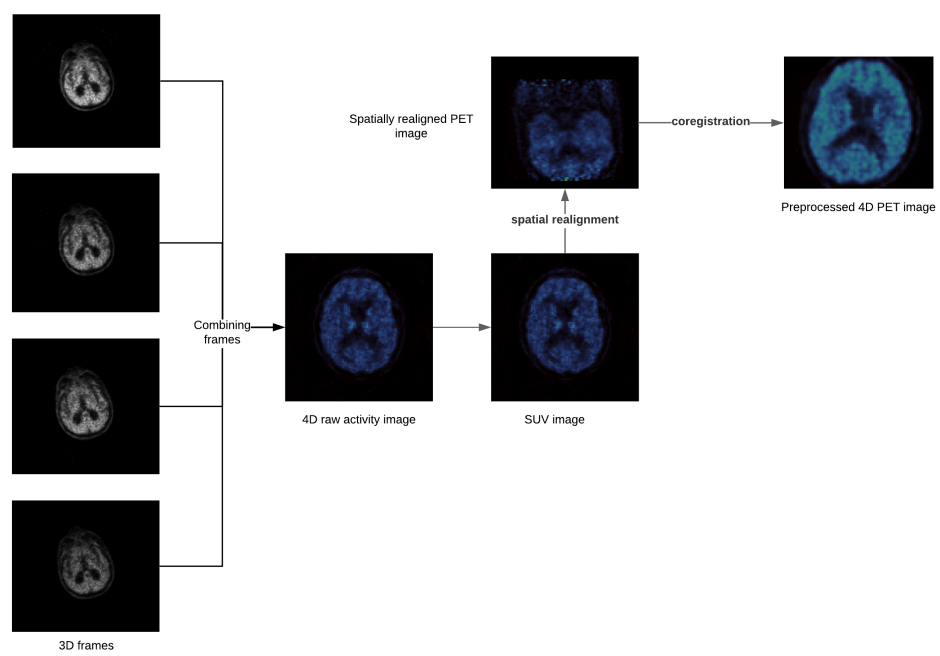

Figure 2. PET image preprocessing flowchart.

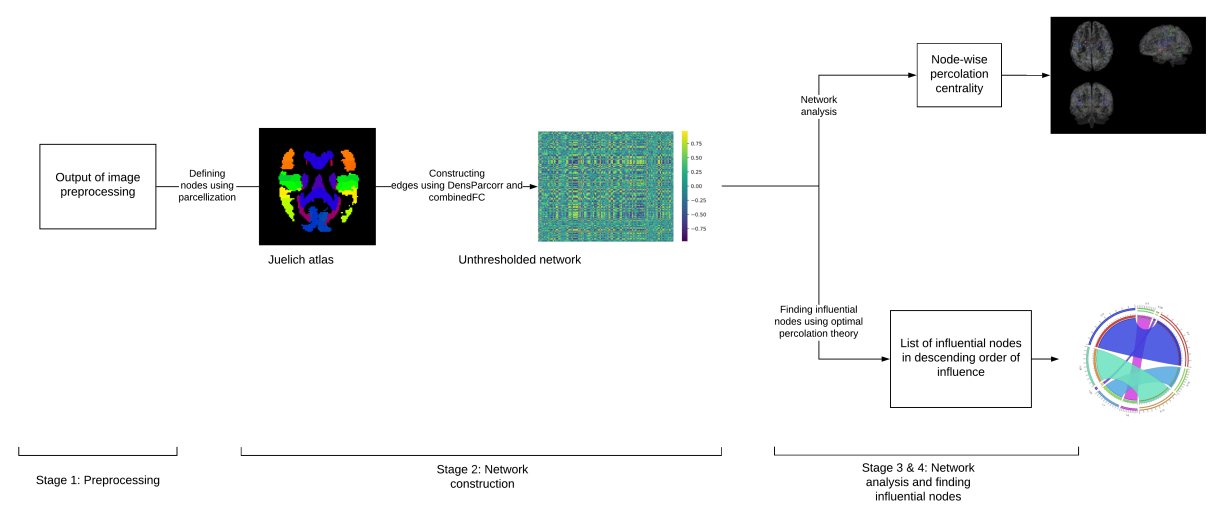

Figure 3. Analysis Pipeline
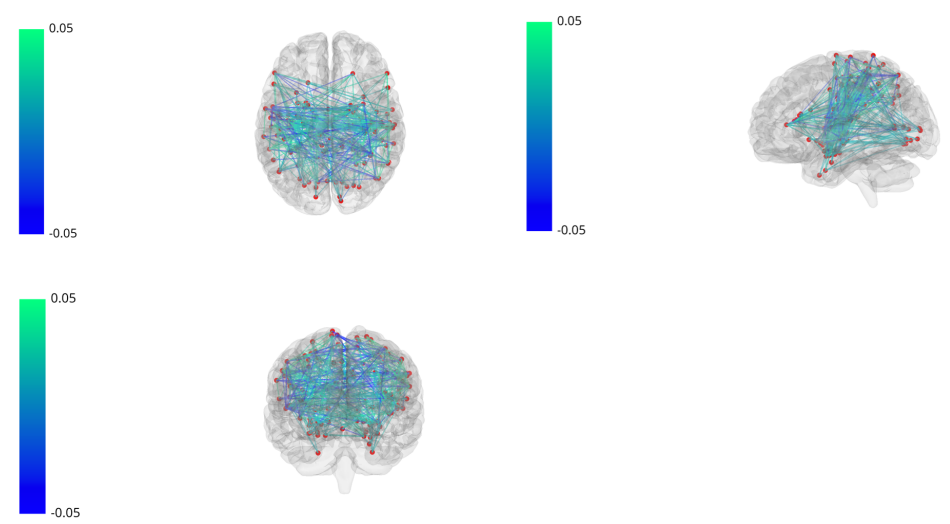

Figure 4. A connected network of all the nodes using the Julich Atlas. Green circles indicate the ROIs, the connecting lines indicate the edges with their weights as denoted by the accompanying color bar 
medRxiv preprint doi: https://doi.org/10.1101/2020.10.12.20211607; this version posted August 3, 2021. The copyright holder for this preprint (which was not certified by peer review) is the author/funder, who has granted medRxiv a license to display the preprint in

It is made available under a CC-BY-NC-ND 4.0 International license .
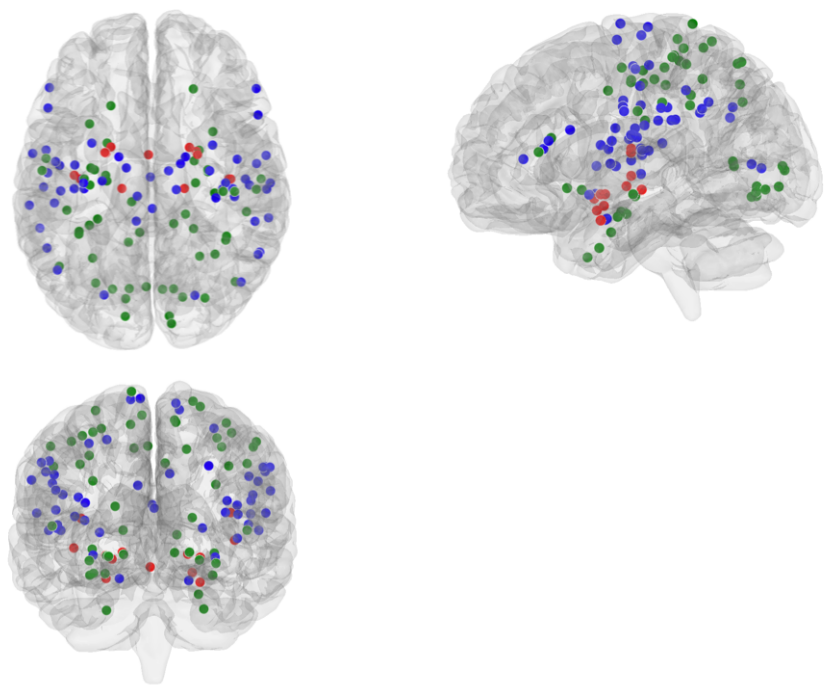

Figure 5. Illustrates the ROIs that corresponds to MMSE and NPIQ. The green circles represent ROIs associated with the MMSE psychometric assessment, the red circles represent ROIs associated with the NPIQ psychometric assessment, and the blue circles represent ROIs associated with both MMSE and NPIQ
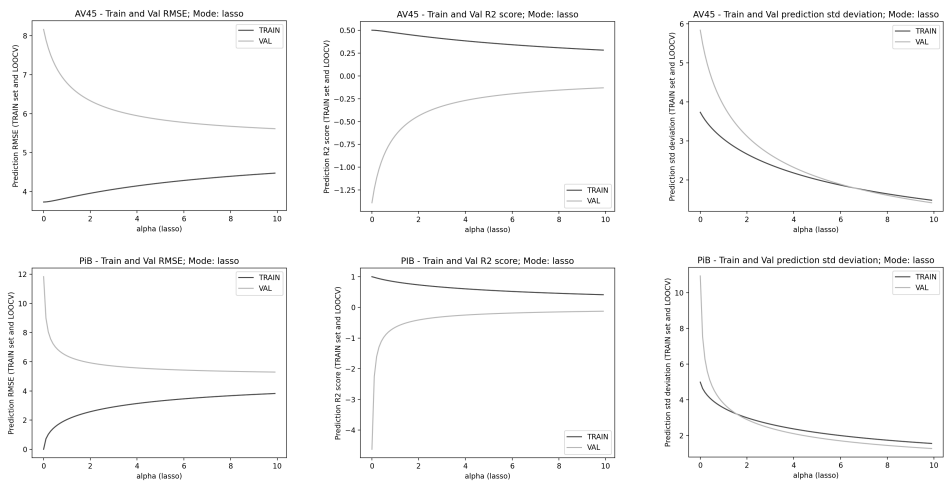

Figure 6. Regularization using Lasso regression with L1 penalty.

Table 1: Distribution of patients

\begin{tabular}{|c|c|c|c|c|c|}
\hline \multicolumn{2}{|c|}{ CN } & \multicolumn{2}{c|}{ MCI } & \multicolumn{2}{c|}{ AD } \\
\hline AV45 & PiB & AV45 & PiB & AV45 & PiB \\
\hline 262 & 13 & 76 & 65 & 116 & 19 \\
\hline M - 122 & M - 8 & M - 54 & M - 45 & M - 67 & M - 11 \\
F - 140 & F - 5 & F - 22 & F - 20 & F - 49 & F - 8 \\
\hline \multicolumn{2}{|l}{ Total - 275; M - 130, F - 145 } & \multicolumn{2}{l|}{ Total - 141; M - 99, F - 42 } & \multicolumn{2}{c|}{ Total - 135; M - 78, F - 57 } \\
\hline
\end{tabular}

Table 2: Number of Scans per tracer type and corresponding critical F-values

\begin{tabular}{|l|c|c|}
\hline Tracer & AV45 & PiB \\
\hline No. of scans & 454 & 97 \\
\hline Critical F-value & 3.002 & 3.027 \\
\hline
\end{tabular}


medRxiv preprint doi: https://doi.org/10.1101/2020.10.12.20211607; this version posted August 3, 2021. The copyright holder for this preprint (which was not certified by peer review) is the author/funder, who has granted medRxiv a license to display the preprint in

It is made available under a CC-BY-NC-ND 4.0 International license .

Table 3: Pairwise ANOVA for AV45 \& PiB tracers for Betweenness Centrality

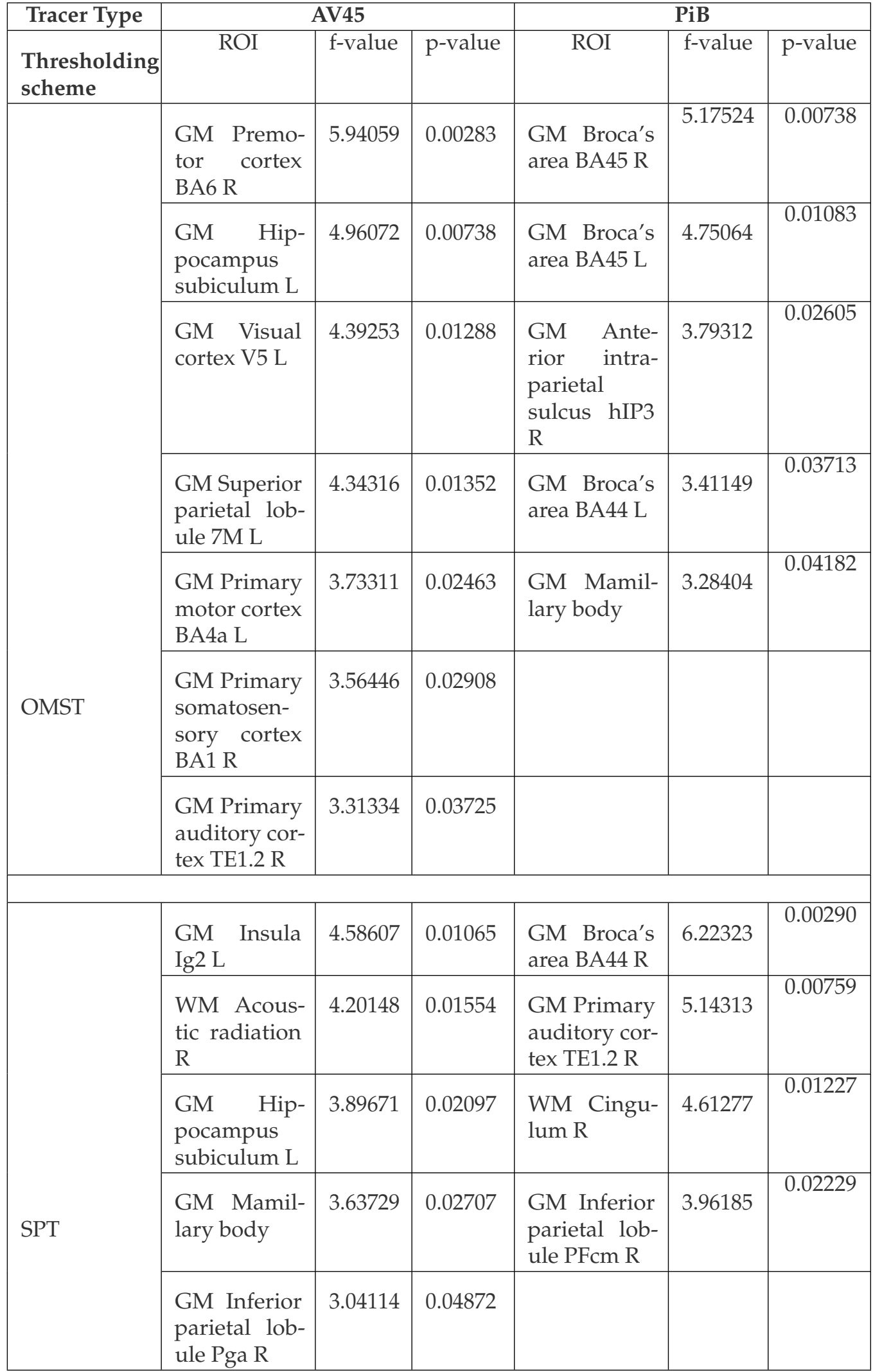


medRxiv preprint doi: https://doi.org/10.1101/2020.10.12.20211607; this version posted August 3, 2021. The copyright holder for this preprint (which was not certified by peer review) is the author/funder, who has granted medRxiv a license to display the preprint in It is made available under a CC-BY-NC-ND 4.0 International license

Table 4: Pairwise ANOVA for AV45 \& PiB tracers for Percolation Centrality

\begin{tabular}{|c|c|c|c|c|c|c|}
\hline Tracer Type & \multicolumn{3}{|c|}{ AV45 } & \multicolumn{3}{|c|}{ PiB } \\
\hline & $\mathrm{ROI}$ & f-value & $\mathrm{p}$-value & ROI & $\mathrm{f}$-value & $\mathrm{p}$-value \\
\hline scheme & $\begin{array}{l}\text { GM Premotor } \\
\text { cortex BA6 R }\end{array}$ & 5.87528 & 0.00302 & $\begin{array}{l}\text { GM Broca's } \\
\text { area BA45 R }\end{array}$ & 5.14527 & 0.00758 \\
\hline & $\begin{array}{l}\text { GM Hip- } \\
\text { pocampus } \\
\text { subiculum L }\end{array}$ & 4.85879 & 0.00815 & $\begin{array}{l}\text { GM Broca's } \\
\text { area BA45 L }\end{array}$ & 4.82934 & 0.01008 \\
\hline & $\begin{array}{l}\text { GM Superior } \\
\text { parietal lobule } \\
7 \mathrm{M} \mathrm{L}\end{array}$ & 4.48843 & 0.01172 & $\begin{array}{l}\text { GM Anterior } \\
\text { intra-parietal } \\
\text { sulcus hIP3 R }\end{array}$ & 3.87541 & 0.02414 \\
\hline & $\begin{array}{l}\text { GM Visual } \\
\text { cortex V5 L }\end{array}$ & 4.38407 & 0.01299 & $\begin{array}{l}\text { GM Broca's } \\
\text { area BA44 L }\end{array}$ & 3.42666 & 0.03661 \\
\hline & $\begin{array}{l}\text { GM Anterior } \\
\text { intra-parietal } \\
\text { sulcus hIP1 L }\end{array}$ & 4.14044 & 0.01650 & $\begin{array}{l}\text { GM Mamil- } \\
\text { lary body }\end{array}$ & 3.33030 & 0.04005 \\
\hline \multirow{4}{*}{ OMST } & $\begin{array}{l}\text { GM Superior } \\
\text { parietal lobule } \\
7 \mathrm{P} \mathrm{R}\end{array}$ & 3.83170 & 0.02235 & & & \\
\hline & $\begin{array}{l}\text { GM Primary } \\
\text { motor cortex } \\
\text { BA4a L }\end{array}$ & 3.77499 & 0.02364 & & & \\
\hline & $\begin{array}{l}\text { GM Primary } \\
\text { somatosen- } \\
\text { sory cortex } \\
\text { BA1 R }\end{array}$ & 3.65131 & 0.02670 & & & \\
\hline & $\begin{array}{l}\text { GM Primary } \\
\text { auditory cor- } \\
\text { tex TE1.2 R }\end{array}$ & 3.34477 & 0.03611 & & & \\
\hline \multirow{6}{*}{ SPT } & $\begin{array}{l}\text { GM Insula Ig2 } \\
\text { L }\end{array}$ & 4.64660 & 0.01004 & $\begin{array}{l}\text { GM Broca's } \\
\text { area BA44 R }\end{array}$ & 6.30152 & 0.00270 \\
\hline & $\begin{array}{l}\text { WM Acoustic } \\
\text { radiation } R\end{array}$ & 4.38388 & 0.01299 & $\begin{array}{l}\text { GM Primary } \\
\text { auditory cor- } \\
\text { tex TE1.2 R }\end{array}$ & 5.03631 & 0.00836 \\
\hline & $\begin{array}{l}\text { GM Hip- } \\
\text { pocampus } \\
\text { subiculum L }\end{array}$ & 3.93752 & 0.02014 & $\begin{array}{l}\text { WM Cingu- } \\
\operatorname{lum} R\end{array}$ & 4.57068 & 0.01275 \\
\hline & $\begin{array}{l}\text { GM Mamil- } \\
\text { lary body }\end{array}$ & 3.54618 & 0.02961 & $\begin{array}{l}\text { GM Inferior } \\
\text { parietal lobule } \\
\text { PFcm R }\end{array}$ & 3.94533 & 0.02263 \\
\hline & $\begin{array}{l}\text { GM Inferior } \\
\text { parietal lobule } \\
\text { Pga R }\end{array}$ & 3.17572 & 0.04266 & & & \\
\hline & $\begin{array}{l}\text { GM Lateral } \\
\text { geniculate } \\
\text { body L }\end{array}$ & 3.05960 & 0.04784 & & & \\
\hline
\end{tabular}


Table 5: Distribution of ROI's across graph metrics and tracer type on the basis of ANOVA test.

\begin{tabular}{lcccc}
\hline $\begin{array}{l}\text { Threshold } \\
\text { Scheme }\end{array}$ & \multicolumn{2}{c}{ OMST } & \multicolumn{2}{c}{ SPT } \\
\hline $\begin{array}{l}\text { Tracer/ } \\
\text { Centrality Measure }\end{array}$ & AV45 & PiB & AV45 & PiB \\
BC & 9 & 5 & 5 & 4 \\
CC & 33 & 29 & 10 & 6 \\
CFBC & 5 & 2 & 1 & 12 \\
EVC & 4 & 11 & 9 & 9 \\
PC & 9 & 5 & 6 & 4 \\
\hline
\end{tabular}

Table 6: Multivariate Linear Regression Analysis- Number of Region of Interest Across Clinical Conditions for Both Threshold Schemes for Betweenness Centrality

\begin{tabular}{|c|c|c|c|c|c|c|c|c|}
\hline \multicolumn{2}{|c|}{ Clinical Condition } & \multicolumn{2}{c|}{ CN } & \multicolumn{2}{c|}{ MCI } & \multicolumn{2}{c|}{ AD } \\
\hline Graph Metric & PT & Tracer & OMST & SPT & OMST & SPT & OMST & SPT \\
\hline \multirow{3}{*}{ Betweenness Centrality } & \multirow{2}{*}{ MMSE } & AV45 & 4 & 5 & 13 & 8 & 4 & 7 \\
\cline { 2 - 9 } & & PiB & 5 & 4 & 5 & 1 & 2 & 9 \\
\cline { 2 - 9 } & \multirow{2}{*}{ NPI-Q } & AV45 & 0 & 3 & 1 & 1 & 0 & 0 \\
\cline { 3 - 9 } & & PiB & 2 & 14 & 1 & 0 & 3 & 10 \\
\hline
\end{tabular}

Table 7: Multivariate Linear Regression Analysis- Number of Region of Interest Across Clinical Conditions for Both Threshold Schemes for Percolation Centrality

\begin{tabular}{|c|c|c|c|c|c|c|c|c|}
\hline & \multicolumn{2}{c|}{ Clinical Condition } & \multicolumn{2}{c|}{ CN } & \multicolumn{2}{c|}{ MCI } & \multicolumn{2}{c|}{ AD } \\
\hline Graph Metric & PT & Tracer & OMST & SPT & OMST & SPT & OMST & SPT \\
\hline \multirow{4}{*}{ Percolation Centrality } & \multirow{2}{*}{ MMSE } & AV45 & 4 & 6 & 12 & 2 & 4 & 7 \\
\cline { 2 - 9 } & & PiB & 5 & 3 & 5 & 0 & 2 & 9 \\
\cline { 2 - 9 } & \multirow{2}{*}{ NPI-Q } & AV45 & 6 & 3 & 2 & 1 & 0 & 0 \\
\cline { 3 - 9 } & & PiB & 2 & 14 & 0 & 1 & 3 & 10 \\
\hline
\end{tabular}

PT: Psychometric Assessment, OMST: Orthogonal Minimum Spanning Tree, SPT: Shortest Path Threshold, MMSE: Mini Mental State Examination, NPIQ: Neuropsychiatric Inventory Questionnaire Questionnaire

\section{Discussion}

Here, a comparison of five nodal metrics; Betweenness centrality, closeness centrality, current flow betweenness centrality, Eigenvector centrality, and percolation centrality, is carried out to better understand the study. Based on the variance analysis and multivariate regression testing and the percolation centrality graph metric computed using the PET images, it is possible to show Alzheimer's disease progressing through the beta-amyloid/tau protein networks.

The student $t$-test provides nodes for each of the five centrality measures across the three clinical conditions, tracer types, and threshold schemes. Here, only the Current Flow Betweenness Centrality Measure fails to provide ROIs across all conditions (see tables 6 through 10), provides only three in cognitively normal condition and two in a mild cognitively impaired condition, both using the OMST scheme for threshold.

It is observed that percolation centrality values of certain areas of the brain, such as inferior and superior parietal lobules, are reliable for the tracer PiB. In contrast, for most other cases, the brain areas differ for each tracer considerably. The variation due 
to the tracers could be because AV45 and PiB bind to the amyloids differently. It is also observed that the percolation centrality of Broca's area is a reliable differentiator between C.N. and A.D. clinical conditions, which validates previous findings that cognitive impairment affects speech production[54].

The MLR analysis for each of the centrality measures across the clinical conditions for both the tracer types provides, on average, one to two ROIs across conditions that can be considered as markers for studying Alzheimer's disease. Expect the current flow betweenness centrality measure, which performed poorly in this study providing only four(3-CN, 1-MCI) ROIs for both the threshold schemes and only one(MCI) ROI for AV45 and PiB tracers, respectively.

Further, when MLR is carried out for the NPI questionnaire, it provides fewer ROIs for each of the centrality measures. When comparing the contribution of ROIs for MMSE-related tasks and NPIQ related tasks. Percolation centrality has the highest percentage of $41 \%$ of ROIs. Followed by Closeness centrality with $40.5 \%$, Betweenness centrality with $30 \%$.

The results from the Scheffe test provide a means to validate and increase the confidence in the results- the leave one out cross-validation (LOOCV) strategy to test the robustness and reliability of our regression. Here cross-validation strategy is implemented because it is unbiased and better suited to our smaller sample size. Using the regularization (L1 - Lasso or L2 - Ridge) to control for overfitting, it is observed that increasing regularization on validation RMSE.

The ROIs obtained from the pairwise t-test for between the clinical conditions show that the OMST scheme provides a higher number of valid ROIs across the five centrality measures. In comparison between the two tracer types and threshold schemes, AV45 provides 92 ROIs, whereas PiB gives 88 ROIs. Of this, $33.7 \%$ and $39.8 \%$ of the ROIs are based on the Shortest Path threshold scheme.

Previous studies show that the seeding of amyloid-beta occurs in neocortical and subcortical regions [55]; from this study, it is observed that for $\mathrm{PiB}$, the following ROI W.M. Superior occipito-frontal fascicle $\mathrm{R}$ is part of both the neocortical and subcortical regions of the brain. Apart from this, AV45 tracer has G.M. Medial geniculate body L ROI in the subcortical region and the following in the neocortical region-G.M. Superior parietal lobule 7P L, G.M. Anterior intra-parietal sulcus hIP3 R, G.M. Superior parietal lobule 7A L, and G.M. Superior parietal lobule 5L L, these are picked up with OMST scheme across all conditions when compared to SPT.

Prior research shows that damage to the parietal lobe is common in A.D., which can lead to apraxia[54,56], which is attested by these results. A.D. is associated with atrophy of the cornu ammonis, the subfield of the hippocampus, and deficits in episodic memory and spatial orientation[57-59]

And the following in the subcortical region - GM Amygdala-laterobasal group L, GM Amygdala-laterobasal group R and GM Hippocampus hippocampal- amygdaloid transition area R. Age factor not so important but the presence of beta amyloid deposits is[60], Since these ROIs stand out irrespective of the clinical condition or demographic backgrounds, the percolation centrality has a potential to be a reliable value for $\mathrm{AD}$ diagnosis, these ROIs are picked up by both tracers and threshold schemes.

Recent methods include genetic and protein markers to improve predicting the course of the disease[61], Genetic testing[62]for markers of A.D., the apolipoprotein-e4 (APOE-e4)[63], or the use of blood testing or brain imaging to rule out dementia due to other factors. These methods rely on many data points and equally reliable computing hardware; this is currently a challenge.

Given that A.D. diagnosis is a global challenge, a method that works well in a spectrum of nations, from developed countries such as the United States to rural hospitals of southeast Asia or Africa[2] is necessary. Methods such as principal component analysis have a few drawbacks; for instance, choosing the number of principal components and data standardization for multiple PET scans of patients with different tracers leads 
to controlling multiple variables. Or using regression analysis which is based on the assumption that there are cause and effect in place. Furthermore, a relationship that is present within a limited data set might get overturned with a detailed data set.

\subsubsection{Limitations}

This study does not give any evidence regarding the disease progression in terms of the ROIs or patient clinical group. However, this can be addressed by increasing the number of observations within each patient clinical group.

The PET tracers used for acquiring the images, Pittsburgh Compound B (PiB) and Florbetapir (AV45), are compared to check for which among the two tracers provide a more consistent or reliable PCv. Here, the AV45 tracer binds with a high affinity to the beta-amyloid plaque, whereas $\mathrm{PiB}$ binds to oligomers or protofibrils. A possible explanation for the difference in $\mathrm{PCv}$ generated using these tracers would be their binding targets. The use of second-generation tracers can help improve the accuracy and test the applicability of percolation centrality on other neurodegenerative diseases and the possibility of using it in metastatic cancer scenarios.

Expanding the dataset to include more patients and comprehensive data that factors in healthy aging shrinkage of the brain, which results in a decrease of the distances of the brain networks, can help improve the reliability of the percolation centrality value. This can then provide a setting for testing out other psychological assessments that can be used as early indicators for dementia due to Alzheimer's disease, thereby tailoring it to specific demographics or population subsets.

The current pipeline is built for tracers such as AV45 and PiB, which indicate betaamyloid plaque concentrations directly and as a post-hoc implementation. However, the pipeline can work with second-generation tracers and tracers like FDG with some appropriate modifications, namely: taking the multiplicative inverse of the percolation states of each of the ROIs to reflect the behavior of the FDG tracer.

A comparison of the ROIs across clinical conditions and tracers does not provide any new information at this stage regarding a common or group of common ROIs across the data(see Table 6. and 7.).

\section{Conclusions}

This study shows that percolation centrality is a reliable predictor and identifies the nodes that regulate the movement of beta-amyloid plaque and use them to track the disease.

This work demonstrates that using the existing neuroimaging method, PET-CT, can add value with relatively short computation time provided sufficient hardware capability is present. The ability to provide a metric to the extent of the disease state is advantageous to the current world of Alzheimer's. Prolonging life with modern-day medicine pushes patients to a world of medical experiences that deviate from the normal. Being able to show the deviation with a value such as percolation centrality has potential applications.

The reliability of percolation centrality can be improved by addressing the concerns that arise by the factors such as the number of patients and the number of patients within each clinical group, time points of data collection, demographics, and the PET tracers used were the limiting factors. Thus, this study provides the usability of percolation centrality value to determine the patient's state and sets the stage for studying other neurodegenerative diseases.

Unlike measures such as hub centrality or betweenness centrality, which provide information regarding a vital vertex/node within a network, the collective influence algorithm provides a minimum set of nodes of the network that are key to the beta-amyloid plaque movement, which can provide information regarding a particular pathway that is susceptible to the neuropathology. 
The threshold schemes implemented in this study indicate that a data-driven approach such as the Orthogonal Minimum Spanning Tree provides better results compared to the Shortest Path approach. Finally, we rank the ROIs based on influence in the network using the $\mathrm{CI}$ algorithm. We compare the results on two thresholding approaches, SPT and OMST, along with finding the influential nodes; this will help us gauge the reliability across different threshold schemes. CI algorithm gives a ranked list of influential nodes for each network (or each scan).

The rank of a node is then further calculated for each category as the sum of individuals ranks of that node for every scan-wise list, divided by the number of scans it occurs in. Nodes are then ranked accordingly in a given category. This provides a general ranking of nodes in a category $(\mathrm{AD} / \mathrm{MCI} / \mathrm{CN})$ instead of looking at influential nodes in each scan separately. The results differ slightly based on the thresholding scheme adopted but broadly align with MLR results discussed earlier. Since this is an exploratory study, improving robustness across different thresholding schemes can be a possible future work.

Supplementary Materials: Please follow the link https://www.mdpi.com//1/1/0/https:// github.com/raghavprasad13/ADNI-Projectfor the analysis pipeline code, and follow this link https:/ /www.mdpi.com//1/1/0/https://drive.google.com/file/d/1ZIVb6 TFyJt68wb_N8mWgBr3xuhOcdZem/view?usp=sharing for Tables 11 through 18.

Author Contributions: Conceptualization, Gautam and Veeky; methodology, Raghav and Gautam; validation, Gautam, Raghav and Pranav; formal analysis, Gautam, Raghav and Pranav; resources, Veeky Baths; data curation, Gautam and Raghav; writing_original draft preparation, Gautam; writing - review and editing, Gautam, Raghav, Pranav and Veeky; visualization, Raghav and Gautam; supervision, Veeky Baths; All authors have read and agreed to the published version of the manuscript.

Funding: This research received no funding.

Institutional Review Board Statement: Institutional Review Board Waiver Statement- Informed consent from the patients is obtained before the assessment carried out by the ADNI study team (See ADNI website for details), and this study is a secondary data analysis of the ADNI data collection, which aims at providing a simplified metric to an already diagnosed patient. The data access and usage is within the ADNI data use agreements.

Informed Consent Statement: Informed consent was obtained from all patients involved in the study by ADNI study Team(s).

Data Availability Statement: PET images from ADNI database are used in this study.

Conflicts of Interest: The authors declare no conflict of interest.

\section{Abbreviations}

The following abbreviations are used in this manuscript: 
AD Alzheimer Disease

ANOVA Analysis of Variance

AV45 Florbetapir (18F- AV-45)

CI Collective Influence

$\mathrm{CN}$ Cognitively Normal

CSF Cerebrospinal Fluid

DOF Degrees of Freedom

EEG Electroencephalography

FAB Frontal Assessment Battery

fMRI functional Magnetic Resonance Imaging

FSL FMRIB Software Library

542 GNU

$\mathrm{PCv} \quad$ Percolation Centrality Value

PET Positron Emission tomography

PiB Pittsburgh compound B (11C-PIB)

RMSE Root Mean Square Error

MCI Mild Cognitive Impairment

MEG Magnetoencephalography

MLR Multivariate Linear Regression

MMSE Mini-Mental State Examination

MST Minimum Spanning Tree

NPIQ Neuropsychiatric Inventory Questionnaire

OMST Orthogonal Minimum Spanning Tree

\section{Appendix A.}

\section{Appendix A.1. Percolation Centrality Computation}

The percolation centrality value is calculated for each network using the inbuilt function of Networkx. This has a worst-case time complexity of $\mathrm{O}\left(\mathrm{n}^{3}\right)$, where $\mathrm{n}$ is the number of nodes in the network. Using a modified form of Brandes' fast algorithm for betweenness centrality, the complexity can be reduced to $O(\mathrm{~nm})$, where $\mathrm{m}$ is the number of edges. However, percolation centrality calculation with target nodes cannot take advantage of this optimization and has a worst-case time complexity of $\mathrm{O}\left(\mathrm{n}^{3}\right)$

\section{Appendix A.2. Other Graph Metrics-Tables}

Table 8: Multivariate Linear Regression Analysis- Region of Interest Across Clinical Conditions for Both Threshold Schemes for Current Flow Betweenness Centrality

\begin{tabular}{|c|c|c|c|c|c|c|c|c|}
\hline \multicolumn{3}{|c|}{ Clinical Condition } & \multicolumn{2}{|c|}{$\mathrm{CN}$} & \multicolumn{2}{|c|}{ MCI } & \multicolumn{2}{|c|}{$\mathrm{AD}$} \\
\hline Graph Metric & PT & Tracer & OMST & SPT & OMST & SPT & OMST & SPT \\
\hline \multirow{4}{*}{$\begin{array}{c}\text { Current Flow } \\
\text { Betweenness } \\
\text { Centrality }\end{array}$} & \multirow{2}{*}{ MMSE } & AV45 & 3 & 0 & 1 & 0 & 0 & 0 \\
\hline & & $\mathrm{P}_{1}$ & 0 & 0 & 1 & 0 & 0 & 0 \\
\hline & \multirow{2}{*}{ NPI-Q } & AV45 & 0 & 0 & 0 & 0 & 0 & 0 \\
\hline & & $\mathrm{PiB}$ & 0 & 0 & 0 & 0 & 0 & 0 \\
\hline
\end{tabular}

Table 9: Multivariate Linear Regression Analysis- Region of Interest Across Clinical Conditions for Both Threshold Schemes for Eigenvector Centrality

\begin{tabular}{|c|c|c|c|c|c|c|c|c|}
\hline \multicolumn{2}{|c|}{ Clinical Condition } & \multicolumn{2}{c|}{ CN } & \multicolumn{2}{c|}{ MCI } & \multicolumn{2}{c|}{ AD } \\
\hline Graph Metric & PT & Tracer & OMST & SPT & OMST & SPT & OMST & SPT \\
\hline \multirow{3}{*}{$\begin{array}{c}\text { Eigenvector } \\
\text { Centrality }\end{array}$} & \multirow{2}{*}{ MMSE } & AV45 & 2 & 2 & 11 & 4 & 7 & 10 \\
\cline { 2 - 9 } & & PiB & 2 & 2 & 6 & 6 & 8 & 3 \\
\cline { 2 - 9 } & \multirow{2}{*}{ NPI-Q } & AV45 & 1 & 4 & 1 & 1 & 0 & 0 \\
\cline { 3 - 9 } & & PiB & 3 & 0 & 3 & 3 & 2 & 9 \\
\hline
\end{tabular}


medRxiv preprint doi: https://doi.org/10.1101/2020.10.12.20211607; this version posted August 3, 2021. The copyright holder for this preprint (which was not certified by peer review) is the author/funder, who has granted medRxiv a license to display the preprint in

It is made available under a CC-BY-NC-ND 4.0 International license .

Table 10: Multivariate Linear Regression Analysis- Region of Interest Across Clinical Conditions for Both Threshold Schemes for Closeness Centrality

\begin{tabular}{|c|c|c|c|c|c|c|c|c|}
\hline & \multicolumn{2}{c|}{ Clinical Condition } & \multicolumn{2}{c|}{ CN } & \multicolumn{2}{c|}{ MCI } & \multicolumn{2}{c|}{ AD } \\
\hline Graph Metric & PT & Tracer & OMST & SPT & OMST & SPT & OMST & SPT \\
\hline \multirow{3}{*}{ Closeness Centrality } & \multirow{2}{*}{ MMSE } & AV45 & 0 & 5 & 1 & 2 & 2 & 1 \\
\cline { 2 - 9 } & & PiB & 2 & 4 & 46 & 0 & 6 & 0 \\
\cline { 2 - 9 } & \multirow{2}{*}{ NPI-Q } & AV45 & 1 & 0 & 0 & 2 & 0 & 0 \\
\cline { 3 - 9 } & & PiB & 3 & 30 & 1 & 0 & 0 & 10 \\
\hline
\end{tabular}

552 PT: Psychometric Assessment, OMST: Orthogonal Minimum Spanning Tree, SPT: Shortest Path Thresh553 old, MMSE: Mini Mental State Examination, NPIQ: Neuropsychiatric Inventory Questionnaire Questionnaire

\section{References}

1. $\quad$ El-Hayek, Y.H.; Wiley, R.E.; Khoury, C.P.; Daya, R.P.; Ballard, C.; Evans, A.R.; Karran, M.; Molinuevo, J.L.; Norton, M.; Atri, A. Tip of the Iceberg: Assessing the Global Socioeconomic Costs of Alzheimer's Disease and Related Dementias and Strategic Implications for Stakeholders. Journal of Alzheimer's Disease 2019, 70, 1-19. doi:10.3233/jad-190426.

2. 2020 Alzheimer's disease facts and figures. Alzheimer's and Dementia 2020, 16, 391-460. doi:10.1002/alz.12068.

3. Johnson, K.A.; Minoshima, S.; Bohnen, N.I.; Donohoe, K.J.; Foster, N.L.; Herscovitch, P.; Karlawish, J.H.; Rowe, C.C.; Carrillo, M.C.; Hartley, D.M.; Hedrick, S.; Pappas, V.; Thies, W.H. Appropriate use criteria for amyloid PET: a report of the Amyloid Imaging Task Force, the Society of Nuclear Medicine and Molecular Imaging, and the Alzheimer's Association. Alzheimer's $\mathcal{E}$ dementia : the journal of the Alzheimer's Association 2013, 9, e-1-16. doi:10.1016/j.jalz.2013.01.002.

4. Shaw, L.M.; Arias, J.; Blennow, K.; Galasko, D.; Molinuevo, J.L.; Salloway, S.; Schindler, S.; Carrillo, M.C.; Hendrix, J.A.; Ross, A.; Illes, J.; Ramus, C.; Fifer, S. Appropriate use criteria for lumbar puncture and cerebrospinal fluid testing in the diagnosis of Alzheimer's disease. Alzheimer's E dementia : the journal of the Alzheimer's Association 2018, 14, $1505-1521$. doi:10.1016/j.jalz.2018.07.220.

5. Landau, S.M.; Thomas, B.A.; Thurfjell, L.; Schmidt, M.; Margolin, R.; Mintun, M.; Pontecorvo, M.; Baker, S.L.; Jagust, W.J.; Initiative, A.D.N. Amyloid PET imaging in Alzheimer's disease: a comparison of three radiotracers. European journal of nuclear medicine and molecular imaging 2014, 41, 1398-1407. doi:10.1007/s00259-014-2753-3.

6. Yamin, G.; Teplow, D.B. Pittsburgh Compound-B (PiB) binds amyloid $\beta$-protein protofibrils. Journal of neurochemistry 2017, 140, 210-215. doi:10.1111/jnc.13887.

7. Su, Y.; Flores, S.; Wang, G.; Hornbeck, R.C.; Speidel, B.; Joseph-Mathurin, N.; Vlassenko, A.G.; Gordon, B.A.; Koeppe, R.A.; Klunk, W.E.; Jack Jr., C.R.; Farlow, M.R.; Salloway, S.; Snider, B.J.; Berman, S.B.; Roberson, E.D.; Brosch, J.; Jimenez-Velazques, I.; van Dyck, C.H.; Galasko, D.; Yuan, S.H.; Jayadev, S.; Honig, L.S.; Gauthier, S.; Hsiung, G.Y.R.; Masellis, M.; Brooks, W.S.; Fulham, M.; Clarnette, R.; Masters, C.L.; Wallon, D.; Hannequin, D.; Dubois, B.; Pariente, J.; Sanchez-Valle, R.; Mummery, C.; Ringman, J.M.; Bottlaender, M.; Klein, G.; Milosavljevic-Ristic, S.; McDade, E.; Xiong, C.; Morris, J.C.; Bateman, R.J.; Benzinger, T.L. Comparison of Pittsburgh compound B and florbetapir in cross-sectional and longitudinal studies. Alzheimer's E Dementia: Diagnosis, Assessment $\mathcal{E}$ Disease Monitoring 2019, 11, 180-190, [https://alz-journals.onlinelibrary.wiley.com/doi/pdf/10.1016/j.dadm.2018.12.008]. doi:https://doi.org/10.1016/j.dadm.2018.12.008.

8. Tombaugh, T.N.; McDowell, I.; Kristjansson, B.; Hubley, A.M. Mini-Mental State Examination (MMSE) and the Modified MMSE (3MS): A psychometric comparison and normative data. Psychological Assessment 1996, 8, 48-59. doi:10.1037//1040-3590.8.1.48.

9. Dubois, B.; Slachevsky, A.; Litvan, I.; Pillon, B. The FAB: a Frontal Assessment Battery at bedside. Neurology 2000, 55, 1621-1626. doi:10.1212/wnl.55.11.1621.

10. Cummings, J.L.; Mega, M.; Gray, K.; Rosenberg-Thompson, S.; Carusi, D.A.; Gornbein, J. The Neuropsychiatric Inventory: comprehensive assessment of psychopathology in dementia. Neurology 1994, 44, 2308-2314. doi:10.1212/wnl.44.12.2308.

11. Reijneveld, J.C.; Ponten, S.C.; Berendse, H.W.; Stam, C.J. The application of graph theoretical analysis to complex networks in the brain. Clinical Neurophysiology 2007, 118, 2317-2331. doi:10.1016/j.clinph.2007.08.010.

12. Stam, C.J.; Reijneveld, J.C. Graph theoretical analysis of complex networks in the brain, 2007. doi:10.1186/1753-4631-1-3.

13. Sakkalis, V. Modern electroencephalographic assessment techniques: Theory and applications; 2014; pp. 1-383. doi:10.1007/978-1-49391298-8.

14. Vecchio, F.; Miraglia, F.; Maria Rossini, P. Connectome: Graph theory application in functional brain network architecture. Clinical Neurophysiology Practice 2017, 2, 206-213. doi:10.1016/j.cnp.2017.09.003.

15. de Haan, W.; Mott, K.; van Straaten, E.C.; Scheltens, P.; Stam, C.J. Activity Dependent Degeneration Explains Hub Vulnerability in Alzheimer's Disease. PLoS Computational Biology 2012, 8, [arXiv:10.1371/journal.pcbi.1002582]. doi:10.1371/journal.pcbi.1002582.

16. Tijms, B.M.; Wink, A.M.; de Haan, W.; van der Flier, W.M.; Stam, C.J.; Scheltens, P.; Barkhof, F. Alzheimer's disease: connecting findings from graph theoretical studies of brain networks. Neurobiology of Aging 2013, 34, $2023-2036$. doi:10.1016/j.neurobiolaging.2013.02.020. 
17. Tijms, B.M.; Wink, A.M.; de Haan, W.; van der Flier, W.M.; Stam, C.J.; Scheltens, P.; Barkhof, F. Alzheimer's disease: connecting findings from graph theoretical studies of brain networks. Neurobiology of Aging 2013, 34, $2023-2036$. doi:10.1016/j.neurobiolaging.2013.02.020.

18. Wook Yoo, S.; Han, C.E.; Shin, J.S.; Won Seo, S.; Na, D.L.; Kaiser, M.; Jeong, Y.; Seong, J.K. A network flow-based analysis of cognitive reserve in normal ageing and Alzheimer's disease. Scientific Reports 2015, 5, 1-14. doi:10.1038/srep10057.

19. Chung, J.; Yoo, K.; Kim, E.; Na, D.L.; Jeong, Y. Glucose Metabolic Brain Networks in Early-Onset vs. Late-Onset Alzheimer's Disease , 2016.

20. Son, S.J.; Kim, J.; Seo, J.; min Lee, J.; Park, H. Connectivity analysis of normal and mild cognitive impairment patients based on FDG and PiB-PET images. Neuroscience Research 2015, 98, 50-58. doi:10.1016/j.neures.2015.04.002.

21. Klyuzhin, I.S.; Fu, J.F.; Hong, A.; Sacheli, M.; Shenkov, N.; Matarazzo, M.; Rahmim, A.; Jon Stoessl, A.; Sossi, V. Data-driven, voxel-based analysis of brain PET images: Application of PCA and LASSO methods to visualize and quantify patterns of neurodegeneration. PLOS ONE 2018, 13, 1-20. doi:10.1371/journal.pone.0206607.

22. McKhann, G.M.; Knopman, D.S.; Chertkow, H.; Hyman, B.T.; Jack, C.R.J.; Kawas, C.H.; Klunk, W.E.; Koroshetz, W.J.; Manly, J.J.; Mayeux, R.; Mohs, R.C.; Morris, J.C.; Rossor, M.N.; Scheltens, P.; Carrillo, M.C.; Thies, B.; Weintraub, S.; Phelps, C.H. The diagnosis of dementia due to Alzheimer's disease: recommendations from the National Institute on Aging-Alzheimer's Association workgroups on diagnostic guidelines for Alzheimer's disease. Alzheimer's E dementia : the journal of the Alzheimer's Association 2011, 7, 263-269. doi:10.1016/j.jalz.2011.03.005.

23. Sperling, R.A.; Aisen, P.S.; Beckett, L.A.; Bennett, D.A.; Craft, S.; Fagan, A.M.; Iwatsubo, T.; Jack, C.R.J.; Kaye, J.; Montine, T.J.; Park, D.C.; Reiman, E.M.; Rowe, C.C.; Siemers, E.; Stern, Y.; Yaffe, K.; Carrillo, M.C.; Thies, B.; Morrison-Bogorad, M.; Wagster, M.V.; Phelps, C.H. Toward defining the preclinical stages of Alzheimer's disease: recommendations from the National Institute on Aging-Alzheimer's Association workgroups on diagnostic guidelines for Alzheimer's disease. Alzheimer's E dementia : the journal of the Alzheimer's Association 2011, 7, 280-292. doi:10.1016/j.jalz.2011.03.003.

24. Kötter, R.; Mazziotta, J.; Toga, A.; Evans, A.; Fox, P.; Lancaster, J.; Zilles, K.; Woods, R.; Paus, T.; Simpson, G.; Pike, B.; Holmes, C.; Collins, L.; Thompson, P.; MacDonald, D.; Iacoboni, M.; Schormann, T.; Amunts, K.; Palomero-Gallagher, N.; Geyer, S.; Parsons, L.; Narr, K.; Kabani, N.; Goualher, G.L.; Boomsma, D.; Cannon, T.; Kawashima, R.; Mazoyer, B. A probabilistic atlas and reference system for the human brain: International Consortium for Brain Mapping (ICBM). Philosophical Transactions of the Royal Society of London. Series B: Biological Sciences 2001, 356, 1293-1322. doi:10.1098/rstb.2001.0915.

25. Eickhoff, S.B.; Stephan, K.E.; Mohlberg, H.; Grefkes, C.; Fink, G.R.; Amunts, K.; Zilles, K. A new SPM toolbox for combining probabilistic cytoarchitectonic maps and functional imaging data. NeuroImage 2005, 25, 1325-1335. doi:10.1016/j.neuroimage.2004.12.034.

26. Desikan, R.S.; Ségonne, F.; Fischl, B.; Quinn, B.T.; Dickerson, B.C.; Blacker, D.; Buckner, R.L.; Dale, A.M.; Maguire, R.P.; Hyman, B.T.; Albert, M.S.; Killiany, R.J. An automated labeling system for subdividing the human cerebral cortex on MRI scans into gyral based regions of interest. NeuroImage 2006, 31, 968-980. doi:10.1016/j.neuroimage.2006.01.021.

27. Newman, M.E.; Watts, D.J. Scaling and percolation in the small-world network model. Physical Review E - Statistical Physics, Plasmas, Fluids, and Related Interdisciplinary Topics 1999, 60, 7332-7342, [arXiv:cond-mat/9904419]. doi:10.1103/PhysRevE.60.7332.

28. Piraveenan, M.; Prokopenko, M.; Hossain, L. Percolation Centrality: Quantifying Graph-Theoretic Impact of Nodes during Percolation in Networks. PLOS ONE 2013, 8, e53095.

29. Moore, C.; Newman, M.E.J. Epidemics and percolation in small-world networks. Physical Review E 2000, 61, 5678-5682. doi:10.1103/PhysRevE.61.5678.

30. Newman, M.E.J. Spread of epidemic disease on networks. Physical Review E 2002, 66, 16128. doi:10.1103/PhysRevE.66.016128.

31. Sander, L.M.; Warren, C.P.; Sokolov, I.M.; Simon, C.; Koopman, J. Percolation on heterogeneous networks as a model for epidemics. Mathematical biosciences 2002, 180, 293-305. doi:10.1016/s0025-5564(02)00117-7.

32. Del Ferraro, G.; Moreno, A.; Min, B.; Morone, F.; Pérez-Ramírez, Ú.; Pérez-Cervera, L.; Parra, L.C.; Holodny, A.; Canals, S.; Makse, H.A. Finding influential nodes for integration in brain networks using optimal percolation theory. Nature Communications 2018, 9, [1806.07903]. doi:10.1038/s41467-018-04718-3.

33. Morone, F.; Min, B.; Bo, L.; Mari, R.; Makse, H.A. Collective Influence Algorithm to find influencers via optimal percolation in massively large social media. Scientific reports 2016, 6, 30062. doi:10.1038/srep30062.

34. Chopra, A.; Shan, L.; Eckelman, W.C.; Leung, K.; Latterner, M.; Bryant, S.H.; Menkens, A. Molecular Imaging and Contrast Agent Database (MICAD): evolution and progress. Molecular imaging and biology 2012, 14, 4-13. doi:10.1007/s11307-011-0521-3.

35. Jenkinson, M.; Beckmann, C.F.; Behrens, T.E.J.; Woolrich, M.W.; Smith, S.M. FSL. NeuroImage 2012, 62, 782-790. doi:10.1016/j.neuroimage.2011.09.015.

36. Initiative, A.D.N. PET Acquisition 2017.

37. Jenkinson, M.; Bannister, P.; Brady, M.; Smith, S. Improved Optimization for the Robust and Accurate Linear Registration and Motion Correction of Brain Images. NeuroImage 2002, 17, 825 - 841. doi:https:/ /doi.org/10.1006/nimg.2002.1132.

38. Vizza, P.; Tradigo, G.; Messina, D.; Cascini, G.L.; Veltri, P. Methodologies for the analysis and classification of PET neuroimages. Network Modeling Analysis in Health Informatics and Bioinformatics 2013, 2, 191-208. doi:10.1007/s13721-013-0035-9.

39. Reuter, M.; Schmansky, N.J.; Rosas, H.D.; Fischl, B. Within-subject template estimation for unbiased longitudinal image analysis. NeuroImage 2012, 61, 1402-1418. doi:10.1016/j.neuroimage.2012.02.084. 
medRxiv preprint doi: https://doi.org/10.1101/2020.10.12.20211607; this version posted August 3, 2021. The copyright holder for this

preprint (which was not certified by peer review) is the author/funder, who has granted medRxiv a license to display the preprint in

It is made available under a CC-BY-NC-ND 4.0 International license .

40. Tange, O. GNU Parallel 20200722 ('Privacy Shield') 2020. GNU Parallel is a general parallelizer to run multiple serial command line programs in parallel without changing them., doi:10.5281/zenodo.3956817.

41. Sanchez-Romero, R.; Cole, M.W. Combining multiple functional connectivity methods to improve causal inferences. bioRxiv 2020, [https://www.biorxiv.org/content/early/2020/02/06/841890.full.pdf]. doi:10.1101/841890.

42. Madigan, D. Graphical models in applied multivariate statistics, by J. Whittaker, John Wiley \& Sons, New York, 1990,448 pp. Price: \$59.95. Networks 1994, 24, 125. doi:10.1002/net.3230240213.

43. Kim, S. ppcor: An R Package for a Fast Calculation to Semi-partial Correlation Coefficients. Communications for Statistical Applications and Methods 2015, 22, 665-674. doi:10.5351/CSAM.2015.22.6.665.

44. Dimitriadis, S.I.; Salis, C.; Tarnanas, I.; Linden, D.E. Topological Filtering of Dynamic Functional Brain Networks Unfolds Informative Chronnectomics: A Novel Data-Driven Thresholding Scheme Based on Orthogonal Minimal Spanning Trees (OMSTs) , 2017.

45. Dimitriadis, S.I.; Antonakakis, M.; Simos, P.; Fletcher, J.M.; Papanicolaou, A.C. Data-Driven Topological Filtering Based on Orthogonal Minimal Spanning Trees: Application to Multigroup Magnetoencephalography Resting-State Connectivity. Brain connectivity 2017, 7, 661-670. doi:10.1089/brain.2017.0512.

46. Dimitriadis, S.I.; Laskaris, N.A.; Tsirka, V.; Vourkas, M.; Micheloyannis, S.; Fotopoulos, S. Tracking brain dynamics via timedependent network analysis. Journal of Neuroscience Methods 2010, 193, 145-155. doi:https:/ /doi.org/10.1016/j.jneumeth.2010.08.027.

47. Hagberg, A.A.; Schult, D.A.; Swart, P.J. Exploring Network Structure, Dynamics, and Function using NetworkX. Proceedings of the 7th Python in Science Conference; Varoquaux, G.; Vaught, T.; Millman, J., Eds.; , 2008; pp. 11-15.

48. Bollobás, B.; Bollobás, B.; Riordan, O.; Riordan, O. Percolation; Cambridge University Press, 2006.

49. Morone, F.; Makse, H.A. Influence maximization in complex networks through optimal percolation. Nature 2015, 524, 65-68.

50. Richardson, M.; Domingos, P. Mining Knowledge-Sharing Sites for Viral Marketing. Proceedings of the Eighth ACM SIGKDD International Conference on Knowledge Discovery and Data Mining; Association for Computing Machinery: New York, NY, USA, 2002; KDD '02, p. 61-70. doi:10.1145/775047.775057.

51. Pastor-Satorras, R.; Vespignani, A. Epidemic Spreading in Scale-Free Networks. Phys. Rev. Lett. 2001, 86, $3200-3203$. doi:10.1103/PhysRevLett.86.3200.

52. Wink, A.M. Eigenvector Centrality Dynamics From Resting-State fMRI: Gender and Age Differences in Healthy Subjects. Frontiers in Neuroscience 2019, 13, 648. doi:10.3389/fnins.2019.00648.

53. Wink, A.M.; Tijms, B.M.; ten Kate, M.; Raspor, E.; de Munck, J.C.; Altena, E.; Ecay-Torres, M.; Clerigue, M.; Estanga, A.; Garcia-Sebastian, M.; Izagirre, A.; Martinez-Lage Alvarez, P.; Villanua, J.; Barkhof, F.; Sanz-Arigita, E. Functional brain network centrality is related to APOE genotype in cognitively normal elderly. Brain and Behavior 2018, 8, e01080, [https:/ / onlinelibrary.wiley.com/doi/pdf/10.1002/brb3.1080]. doi:https://doi.org/10.1002/brb3.1080.

54. Fernández, M.; Gobartt, A.L.; Balañá, M.; Group, C.S. Behavioural symptoms in patients with Alzheimer's disease and their association with cognitive impairment. BMC neurology 2010, 10, 87. doi:10.1186/1471-2377-10-87.

55. Jucker, M.; Walker, L.C. Self-propagation of pathogenic protein aggregates in neurodegenerative diseases. Nature 2013, 501, 45-51. doi:10.1038/nature12481.

56. Kueper, J.K.; Speechley, M.; Lingum, N.R.; Montero-Odasso, M. Motor function and incident dementia: a systematic review and meta-analysis. Age and Ageing 2017, 46, 729-738. doi:10.1093/ageing/afx084.

57. Adler, D.H.; Wisse, L.E.; Ittyerah, R.; Pluta, J.B.; Ding, S.L.; Xie, L.; Wang, J.; Kadivar, S.; Robinson, J.L.; Schuck, T.; Trojanowski, J.Q.; Grossman, M.; Detre, J.A.; Elliott, M.A.; Toledo, J.B.; Liu, W.; Pickup, S.; Miller, M.I.; Das, S.R.; Wolk, D.A.; Yushkevich, P.A. Characterizing the human hippocampus in aging and Alzheimer's disease using a computational atlas derived from ex vivo MRI and histology. Proceedings of the National Academy of Sciences of the United States of America 2018, 115, $4252-4257$. doi:10.1073/pnas.1801093115.

58. Masurkar, A.V. Towards a circuit-level understanding of hippocampal CA1 dysfunction in Alzheimer's disease across anatomical axes. Journal of Alzheimer's disease E Parkinsonism 2018, 8, 412.

59. Ogawa, M.; Sone, D.; Beheshti, I.; Maikusa, N.; Okita, K.; Takano, H.; Matsuda, H. Association between subfield volumes of the medial temporal lobe and cognitive assessments. Heliyon 2019, 5, e01828. doi:https://doi.org/10.1016/j.heliyon.2019.e01828.

60. Hamaguchi, T.; Eisele, Y.S.; Varvel, N.H.; Lamb, B.T.; Walker, L.C.; Jucker, M. The presence of A $\beta$ seeds, and not age per se, is critical to the initiation of A $\beta$ deposition in the brain. Acta Neuropathologica 2012, 123, 31-37. doi:10.1007/s00401-011-0912-1.

61. Nichols, E.; Szoeke, C.E.; Vollset, S.E.; Abbasi, N.; Abd-Allah, F.; Abdela, J.; Aichour, M.T.E.; Akinyemi, R.O.; Alahdab, F.; Asgedom, S.W.; Awasthi, A.; Barker-Collo, S.L.; Baune, B.T.; Béjot, Y.; Belachew, A.B.; Bennett, D.A.; Biadgo, B.; Bijani, A.; Bin Sayeed, M.S.; Brayne, C.; Carpenter, D.O.; Carvalho, F.; Catalá-López, F.; Cerin, E.; Choi, J.Y.J.; Dang, A.K.; Degefa, M.G.; Djalalinia, S.; Dubey, M.; Duken, E.E.; Edvardsson, D.; Endres, M.; Eskandarieh, S.; Faro, A.; Farzadfar, F.; Fereshtehnejad, S.M.; Fernandes, E.; Filip, I.; Fischer, F.; Gebre, A.K.; Geremew, D.; Ghasemi-Kasman, M.; Gnedovskaya, E.V.; Gupta, R.; Hachinski, V.; Hagos, T.B.; Hamidi, S.; Hankey, G.J.; Haro, J.M.; Hay, S.I.; Irvani, S.S.N.; Jha, R.P.; Jonas, J.B.; Kalani, R.; Karch, A.; Kasaeian, A.; Khader, Y.S.; Khalil, I.A.; Khan, E.A.; Khanna, T.; Khoja, T.A.; Khubchandani, J.; Kisa, A.; Kissimova-Skarbek, K.; Kivimäki, M.; Koyanagi, A.; Krohn, K.J.; Logroscino, G.; Lorkowski, S.; Majdan, M.; Malekzadeh, R.; März, W.; Massano, J.; Mengistu, G.; Meretoja, A.; Mohammadi, M.; Mohammadi-Khanaposhtani, M.; Mokdad, A.H.; Mondello, S.; Moradi, G.; Nagel, G.; Naghavi, M.; Naik, G.; Nguyen, L.H.; Nguyen, T.H.; Nirayo, Y.L.; Nixon, M.R.; Ofori-Asenso, R.; Ogbo, F.A.; Olagunju, A.T.; Owolabi, M.O.; Panda-Jonas, S.; Passos, V.M.A.; Pereira, D.M.; Pinilla-Monsalve, G.D.; Piradov, M.A.; Pond, C.D.; Poustchi, H.; Qorbani, 
medRxiv preprint doi: https://doi.org/10.1101/2020.10.12.20211607; this version posted August 3, 2021. The copyright holder for this preprint (which was not certified by peer review) is the author/funder, who has granted medRxiv a license to display the preprint in It is made available under a CC-BY-NC-ND 4.0 International license .

M.; Radfar, A.; Reiner, R.C.; Robinson, S.R.; Roshandel, G.; Rostami, A.; Russ, T.C.; Sachdev, P.S.; Safari, H.; Safiri, S.; Sahathevan, R.; Salimi, Y.; Satpathy, M.; Sawhney, M.; Saylan, M.; Sepanlou, S.G.; Shafieesabet, A.; Shaikh, M.A.; Sahraian, M.A.; Shigematsu, M.; Shiri, R.; Shiue, I.; Silva, J.P.; Smith, M.; Sobhani, S.; Stein, D.J.; Tabarés-Seisdedos, R.; Tovani-Palone, M.R.; Tran, B.X.; Tran, T.T.; Tsegay, A.T.; Ullah, I.; Venketasubramanian, N.; Vlassov, V.; Wang, Y.P.; Weiss, J.; Westerman, R.; Wijeratne, T.; Wyper, G.M.; Yano, Y.; Yimer, E.M.; Yonemoto, N.; Yousefifard, M.; Zaidi, Z.; Zare, Z.; Vos, T.; Feigin, V.L.; Murray, C.J. Global, regional, and national burden of Alzheimer's disease and other dementias, 1990-2016: a systematic analysis for the Global Burden of Disease Study 2016. The Lancet Neurology 2019, 18, 88-106. doi:10.1016/S1474-4422(18)30403-4.

62. Loy, C.T.; Schofield, P.R.; Turner, A.M.; Kwok, J.B.J. Genetics of dementia. Lancet (London, England) 2014, 383, 828-840. doi:10.1016/S0140-6736(13)60630-3.

63. Holtzman, D.M.; Herz, J.; Bu, G. Apolipoprotein E and apolipoprotein E receptors: normal biology and roles in Alzheimer disease. Cold Spring Harbor perspectives in medicine 2012, 2, a006312. doi:10.1101/cshperspect.a006312. 

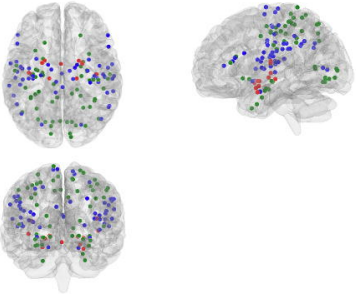


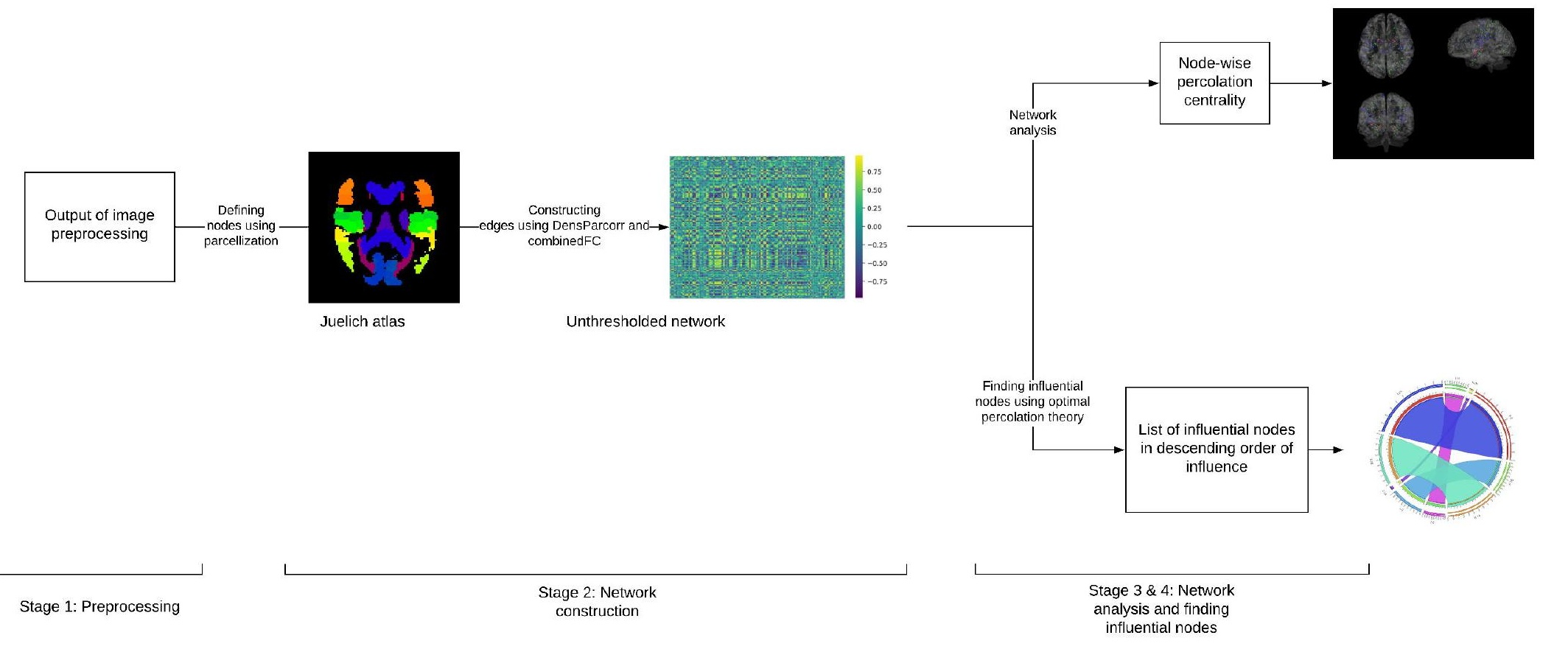



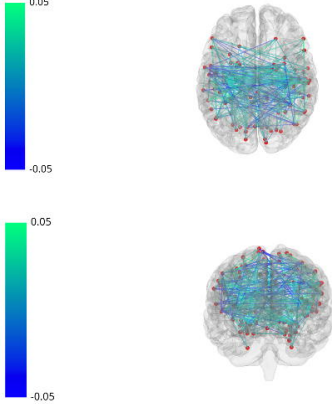


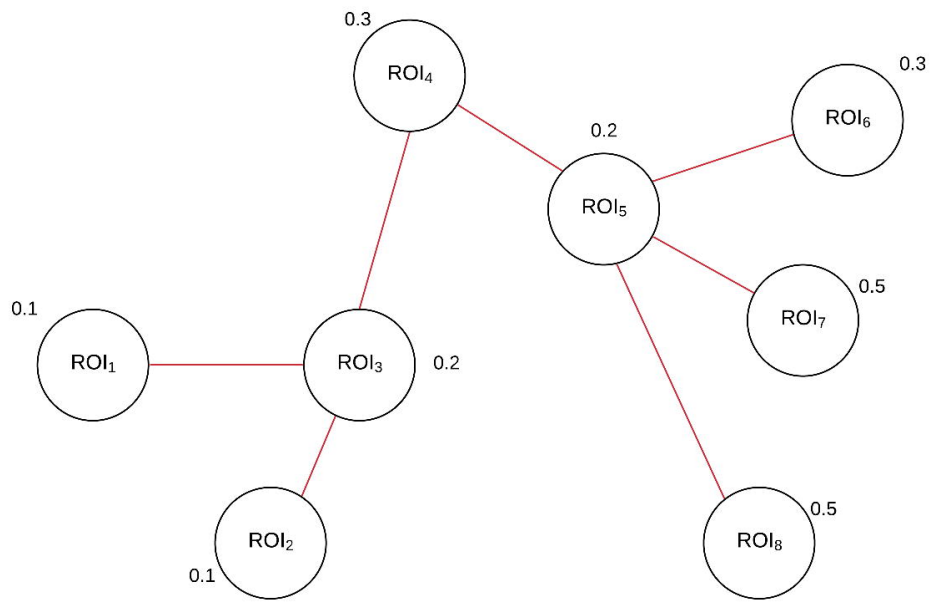

